Family Medicine and Community Health

\section{Exploring the structure of social media application-based information-sharing clinical networks in a community in Japan using a social network analysis approach}

Junji Haruta (D) , ${ }^{1,2}$ Sho Tsugawa, ${ }^{3}$ Kazunari Ogura ${ }^{4}$
To cite: Haruta J, Tsugawa S, Ogura K. Exploring the structure of social media application-based informationsharing clinical networks in a community in Japan using a social network analysis approach. Fam Med Com Health 2020;8:e000396. doi:10.1136/ fmch-2020-000396
Check for updates

(c) Author(s) (or their employer(s)) 2020. Re-use permitted under CC BY-NC. No commercial re-use. See rights and permissions. Published by BMJ.

${ }^{1}$ Medical Education Center, Keio University, Tokyo, Japan

${ }^{2}$ School of Medicine, University of Tsukuba, Tsukuba, Japan ${ }^{3}$ Division of Information Engineering, University of Tsukuba, Tsukuba, Japan ${ }^{4}$ Hachinohe Family Clinic, Hachinohe, Japan

Correspondence to Dr Junji Haruta; junharujp@keio.jp

\section{ABSTRACT}

Objective Currently, use of social networking services (SNSs) for interprofessional collaboration is increasing. However, few studies have reported on virtual interprofessional interactions in community healthcare services. Revealing such structural characteristics of the networks can provide insight into the functions of the interprofessional information-sharing network and lead to smoother collaboration. Thus, we aimed to explore the structure of SNS-based information-sharing clinical networks.

Design Social network analysis (SNA).

Setting We selected a community in City $X$ in Japan. Data collection We analysed SNS-based informationsharing clinical network data linked to patients receiving home medical care or care services between January and December 2018. A network was created for each patient to allow healthcare professionals to post and view messages on the web platform. In the SNA, healthcare professions registered in a patient group were represented as nodes, and message posting/viewing relationships were represented as links in the patient network. We investigated the structural characteristics of the target networks using several measures for SNA, including indegree centrality and outdegree centrality, which reflect the number of incoming and outgoing links to/from a node, respectively. Additionally, the professions forming the most central nodes were investigated based on their ranking to identify those with a central role in the networks. Finally, to compare the networks of nursing care levels 1-3 (lighter care requirement) and those with nursing care levels 4-5 (heavier care requirement), we analysed the structural differences in the networks and investigated the roles of healthcare professionals using centrality measures of nodes.

Results Among 844 groups, 247 groups with any nursing care level data were available for analysis. Increasing nursing care level showed higher density, reciprocity and lower centralisation. Healthcare professions with high indegree centrality (physicians, care workers and physical therapists) differed from those with high outdegree centrality (home care workers, physical therapists, and registered dieticians). Visiting nurses and nurses in the clinic played a central role, but visiting nurses tended to have higher indegree and outdegree centrality, while

\section{Key points}

- Question: To pave the way for smoother collaboration in a community based on multiprofessional network structures, this study aimed to explore the structure of social networking service (SNS)-based information-sharing clinical networks, and the professions that play central roles in community networks using social network analysis (SNA).

- Findings: SNS-based information-sharing networks for healthcare professionals across facilities that provide more home medical care or nursing services had relatively higher density, reciprocity and lower centrality. Healthcare professions with high indegree centrality differed from those with high outdegree centrality. As professionals with central roles in information sharing, visiting nurses had higher indegree and outdegree centrality while nurses in the clinic had higher closeness and betweenness centrality in networks with heavier care requirement.

- Meaning: Given the lack of evidence on SNA in interprofessional collaboration in a community, these findings suggest that SNA has the potential to contribute to improving the provision of patient care and aid in the proposal of highly cost-effective methods. Further studies are needed to determine the relationship between such networks and the successful achievement of good patient outcomes and other factors.

nurses in the clinic had higher closeness and betweenness centrality in networks with heavier care requirement. Conclusion The SNS-based information-sharing clinical network structure showed that different professions played some form of a central role. Associations between network structures and patient outcomes, cost effectiveness and other factors warrant further investigation.

\section{INTRODUCTION}

In Japan's super-ageing society, elderly people encounter an increasing number of physical, psychological and social problems. ${ }^{1}$ These 
problems are complicated by economic and social issues affecting both patients and their families. In addition, the elderly has many and wide-ranging care needs due to complications, comorbidities and conditions unique to this demographic. ${ }^{2}$ It is therefore necessary that healthcare professionals promote and facilitate interprofessional collaboration (IPC). ${ }^{3}$ To achieve this, competency or capability for IPC has been developed in the USA, Canada, Australia and Japan, with communication being a common indispensable factor. ${ }^{4}$ Reviews of interprofessional communication have reported the presence of a relationship between patient outcomes and healthcare communication. ${ }^{56}$ Many studies, however, focus on individual-profession relationships or organisational climates in communication, ${ }^{78}$ whereas studies on clinical network structures are limited.

In a society with increasing complexity, healthcare professionals should seek information sharing via community network systems not only by interprofessional but also intraorganisational communication. In Japan in particular, which is dealing with a super-ageing society, effective clinical interprofessional and intraorganisational networks to improve quality of care and patient outcomes are becoming an urgent need. ${ }^{9}$ Thus, the Japanese government has proposed establishing a communitybased integrated care system by 2025, which comprises a healthcare insurance system and long-term care insurance system for individual communities. The purpose of the long-term care insurance system is to ensure comprehensive medical care, nursing care, preventive care, housing and livelihood support. The system comes into play in healthcare services provided in communities based on patients' needs. The needs of each patient are determined according to the degree of care services required: services are classified into seven levels in accordance with the condition of each patient or user. Care level 5 indicates the highest level of requirement for long-term care, while care level 1 indicates the lowest, and support levels 1 and 2 contain plans for preventing long-term care. ${ }^{10} \mathrm{In}$ recent years, social networking services (SNSs) have been increasingly used by professionals providing home healthcare services to such patients in the community to share patient information. SNS-based information sharing can serve as an effective training tool for education on IPC. ${ }^{11}$ SNSs have enabled the creation of web-based communities and led to improvements in networking and expertise, thus enabling personal dialogues regardless of time, space and geography. ${ }^{12}$ Hence, SNS-based networks are expected to be key contributors to communications among multiprofessionals in community-based integrated systems.

Social network analysis (SNA) is a method for analysing communication patterns among multiple individuals, and is a useful means for healthcare professionals to evaluate interprofessional communication. SNAs have been used since the 1950 s to investigate the social influence of health professionals and technological innovations on the diffusion of information. ${ }^{14}{ }^{15}$ A systematic review of
SNAs in medical practice identified several studies which used SNAs to analyse communication patterns among healthcare professionals. ${ }^{16}$ While most of these studies analysed face-to-face communication processes, ${ }^{17-19}$ some analysed information technology-based communication processes. Studies that analysed social networks of small nursing staff groups, ${ }^{20}$ social networks of surgeons, ${ }^{21}$ and social networks of different profession ${ }^{22-25}$ have reported that they are composed of weak links. Few studies, however, have examined SNS-based networks across healthcare professions providing home medical care and nursing services in the community, a field which will be needed in ageing societies in all developed countries in the future. Identifying the types of network patterns that have been developed among healthcare professionals and the healthcare professions that serve as key players in community networks will be beneficial for community healthcare services. Exploration of SNS-based networks among healthcare professions in a community may illuminate the mechanisms of interprofessional communication patterns. Regarding methods of SNA, questionnaires and interviews are associated with limitations such as recall bias and other factors. We predict that SNS-based networks will become increasingly used as forms of virtual interaction in the near future due to their ability to facilitate networking to enable interactions in the absence of physical contact. Thus, using SNA for objective data visualisation, this study aimed to explore the structure of SNS-based information-sharing clinical networks, and the professions that play central roles in community networks in somewhere in Japan.

\section{METHODS \\ Design}

SNA was performed to analyse communication patterns in SNS-based information-sharing clinical networks in a community. SNA is used to investigate internodal relationships and their influences on the network as a whole based on the principles of graph theory. ${ }^{26}$ SNA examines nodes (eg, individual healthcare professionals) and the existence of internodal links, focusing on node-tonode relationships and link structures (eg, informationsharing clinical networks). In the present study, one interprofessional network was constructed for each patient, on which different professions were allowed to post and view messages using an SNS-based platform. The constructed networks were analysed by quantifying their characteristics and identifying central nodes in the networks.

\section{Setting}

As purposive sampling, we selected a community in City X in Japan because healthcare professionals in that city used an SNS-based platform. City X is a core city in a region with a population of 220000 and 7 general hospitals. 


\section{SNS-based information-sharing tool}

An SNS-based information-sharing tool used in medical practice settings in City X was used in this study. The SNS is created by a private company for use by healthcare professionals. Its users comprise a wide variety of professions, including physicians and nurses, as well as care workers, care managers, physical therapists and registered dieticians. For instance, in home-visit care services, professional staffs provide at patients' home, such as home-based long-term care, visiting nurse, visiting pharmacist, visiting physician and visiting physical or occupational therapists service. Registered dietitians provide nutritional guidance to patients in home or facilities and teach multiprofessionals how to make swallowing diets. Home care workers visit patients' homes to assist with meals, toileting and bathing while care workers assist the residents in the facility. Care managers are responsible for planning care services provided under long-term care insurance. Medical clerks in clinic are responsible for handling reception appointment requests, prescription requests and enquiries and working as part of a small team. Medical consultants are responsible for sharing patient information between facilities, such as when patients are admitted and discharged from the hospital, and for making appointments for social services needed for transportation. In this service, registered healthcare professionals share patient-related information. The service is used by staff members of multiple medical and nursing care facilities.

\section{Data collection}

Data were obtained from patients and healthcare professionals who consented to its anonymised use for research purposes. This study was conducted to investigate patient attributes and information provision/receipt by patients and their respectively linked healthcare professionals who used online message boards in City X between January and December 2018. One group was created per patient. Healthcare professionals registered in the group were allowed to post and view messages on the group's message board. Data analysed included the sex, age and degree of care required for each patient/user participating in the online message board; the professions of the posting users and users who marked 'viewed' on a message; the time stamp included in each message; and the 'degree of care required' for each patient.

\section{Constructing networks}

For each group, a network was constructed from the log data of message postings and viewings for each patient. Individual healthcare professionals (excluding the patient) registered in patient groups were regarded as nodes. Message posting/viewing relationships were regarded as links. More specifically, for each patient group, an unweighted directed graph $G=(V, E)$ was constructed. Node $u$ represents a user (ie, an individual healthcare professional), and directed link $(u, v)$ represents that user $v$ marked 'viewed' on a message posted by user $u$. A link

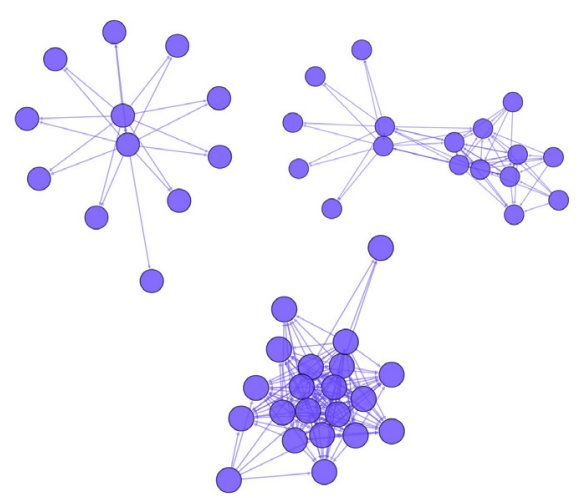

Figure 1 Visualisation of social network patterns.

was therefore considered to have been created when a user made internodal communication via a particular thread. Because networks that are too small are not useful for SNAs, networks with 10 or more nodes were selected for subsequent analysis based on previous studies. ${ }^{27}{ }^{28} \mathrm{~A}$ visualisation of some of the social network patterns in this study is shown in figure 1.

\section{Analysis}

First, we investigated the structural characteristics of the target networks using several measures for SNA. For each network, we mainly obtained the number of nodes, density ${ }^{29}$ reciprocity, ${ }^{30}$ diameter, ${ }^{31}$ path length, ${ }^{32}$ clustering coefficient ${ }^{33}$ and centralisation based on degree, closeness and betweenness. ${ }^{34}$ Density was determined by dividing the number of actual links in the network by the maximum possible number, to provide a more comprehensive explanation of the connectivity level in the network. ${ }^{35}$ Reciprocity is a measure of the percentage of two-way links in a network. ${ }^{30}$ Diameter is the longest shortest path in the network. ${ }^{31}$ The mean path length in a network is defined as the mean distance over all node pairs, including the distance between a node and itself. ${ }^{32}$ The clustering coefficient quantifies the abundance of connected triangles in a network and useful in characterising individual nodes. ${ }^{33}$ Degree centrality is the number of links that a node has. ${ }^{34}$ Closeness and betweenness centralisation are explained below. These measures are widely used in SNAs. ${ }^{27} 28$ All of these measures were normalised to a $(0,1)$ scale and were represented as values ranging from 0 to 1 , and the mean values and SD for each network were calculated. Taking into account the burden of physical and mental care, we compared the median values in groups with nursing care levels 1-3 (lighter care requirement) with those with nursing care levels $4-5$ (heavier care requirement) ${ }^{10}$ using the MannWhitney U test.

Second, we investigated the roles of healthcare professionals using centrality measures of nodes. Centrality measures of each node in each network were calculated. Then, averages of centrality measures were compared among different professions. The centrality measures used in this study were indegree centrality, outdegree centrality, closeness centrality and betweenness centrality, 
all of which are common in SNA. ${ }^{34}$ Indegree centrality and outdegree centrality represent the number of connections of individual nodes. The indegree centrality of a node is defined as the number of incoming links to the node (user who marked 'viewed' on a message), whereas the outdegree centrality of a node is defined as the number of outgoing links from the node (user who posted messages). Closeness centrality of a node is defined as the reciprocal number of the sum of distances from the node to all other nodes, and betweenness centrality is defined as the number of the shortest paths passing through the node.

Finally, we investigated the key player(s) in each group by extracting the most central nodes in the network of the group. Using the four centrality measures (ie, indegree, outdegree and closeness, and betweenness), the nodes with the highest centrality were extracted for each network. Note that multiple nodes were extracted for each network when two or more nodes had the highest centrality. Subsequently, professions of the most central nodes were investigated according to their ranking, and the professions that played central roles in the groups were identified. Additionally, we compared the professions of the central nodes in groups with nursing care levels 1-3 with those with nursing care levels $4-5$.

\section{RESULTS}

The number of groups (patients) with at least one post during the year was 844 . In the SNA, 468 groups with 10 or more nodes were examined, including 247 groups with nursing care level data (figure 2). Node attributes are shown in table 1.

The mean number of nodes was $18.3(\mathrm{SD}=7.5)$. The mean values of network measures were $0.42(\mathrm{SD}=0.15)$ for density, $0.41(\mathrm{SD}=0.2)$ for reciprocity, $4.5(\mathrm{SD}=3.12)$ for diameter, $1.4 \quad(\mathrm{SD}=0.18)$ for path length, 0.81 $(\mathrm{SD}=0.12)$ for clustering coefficient, $0.57 \quad(\mathrm{SD}=0.13)$ for degree-based centralisation, $0.46 \quad(\mathrm{SD}=0.17)$ for

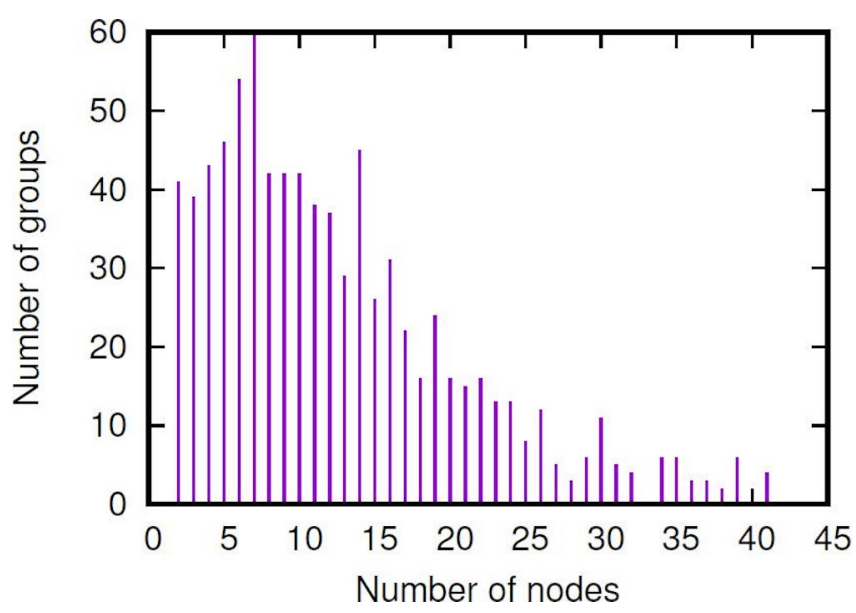

Figure 2 Distribution of groups by the number of nodes (groups with no less than 10 nodes were analysed).
Table 1 Characteristics of patients with 10 or more multiprofessional nodes in home medical care $(n=468)$

\begin{tabular}{|c|c|c|c|}
\hline \multicolumn{4}{|l|}{ Values } \\
\hline \multirow[t]{2}{*}{ Age } & & \multicolumn{2}{|c|}{$80.6(\mathrm{SD}=16.4)$} \\
\hline & & $\mathrm{N}$ & $\%$ \\
\hline \multirow[t]{2}{*}{ Sex } & Men & 176 & 37.6 \\
\hline & Women & 292 & 62.3 \\
\hline \multicolumn{4}{|c|}{$\mathrm{n}=247$ (descriptions available) } \\
\hline Nursing care level 1 & & 27 & 11.0 \\
\hline Nursing care level 2 & & 33 & 13.4 \\
\hline Nursing care level 3 & & 46 & 18.6 \\
\hline Nursing care level 4 & & 62 & 25.1 \\
\hline Nursing care level 5 & & 73 & 29.6 \\
\hline Support level 1 & & 1 & 0.4 \\
\hline Support level 2 & & 5 & 2.0 \\
\hline
\end{tabular}

closeness centralisation and $0.15(\mathrm{SD}=0.16)$ for betweenness centralisation (table 2).

Table 2 shows the results of network characterisation by nursing care level. When nursing care levels 1-3 and 4-5 were compared, the mean number of nodes was $18.8(\mathrm{SD}=7.93)$ and $20.0(\mathrm{SD}=7.91)$, the mean values of network measures were $0.40 \quad(\mathrm{SD}=0.17)$ and 0.44 $(\mathrm{SD}=0.13)$ for density, $0.41(\mathrm{SD}=0.21)$ and $0.44(\mathrm{SD}=0.18)$ for reciprocity, $0.49(\mathrm{SD}=0.19)$ and $0.42(\mathrm{SD}=0.14)$ for closeness centralisation, and $0.16(\mathrm{SD}=0.17)$ and 0.11 $(\mathrm{SD}=0.11)$ for betweenness centralisation. In general, an increase in nursing care level showed a high number of nodes, higher density and reciprocity, and lower centralisation (table 2). Comparison of median differences in various network measures between nursing care levels 1-3 and 4-5 using the Mann-Whitney U-test revealed significant differences $(p<0.05)$ for the measures, except number of nodes (table 2 ).

Next, the data were analysed for differences in the averages of centrality measures among different professions for the top five professions (figure 3). Physicians, care workers and physical therapists tended to have high indegree centrality while home care workers, physical therapists and registered dieticians tended to have high outdegree centrality. Closeness centrality showed little difference among professions whereas betweenness centrality tended to be higher for medical clerks, registered dieticians and physicians (figure 3 ).

On the other hand, figure 4 shows the top-five ranking of the professions who indicated the highest centrality in each network. The top five-ranked professions of the most central nodes were compared by nursing care level (figure 4). Nurses, visiting nurses, care workers and care managers played central roles in many groups. According to differences in nursing care levels, centrality was generally high for visiting nurses and nurses in the clinic in groups with light and heavy care requirement, respectively. In particular, the rank of indegree and outdegree 
Table 2 Overall network structure

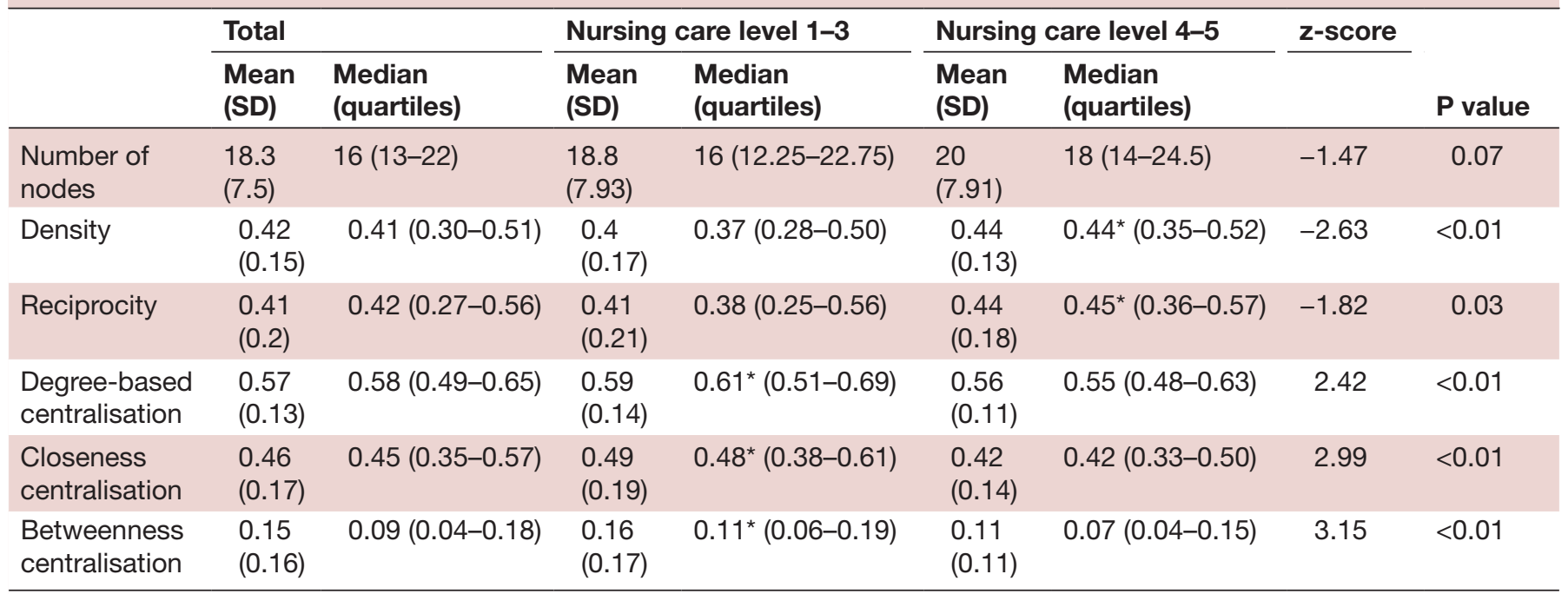

*Mann-Whitney U-test revealed significant differences, $\mathrm{p}<0.05$.

centrality of visiting nurses tended to be higher for heavier care requirement, while the rank of closeness and betweenness centrality of nurses in the clinic tended to be higher than those of visiting nurses.

\section{DISCUSSION}

Using SNA, this study in one community in Japan showed that SNS-based information-sharing networks for

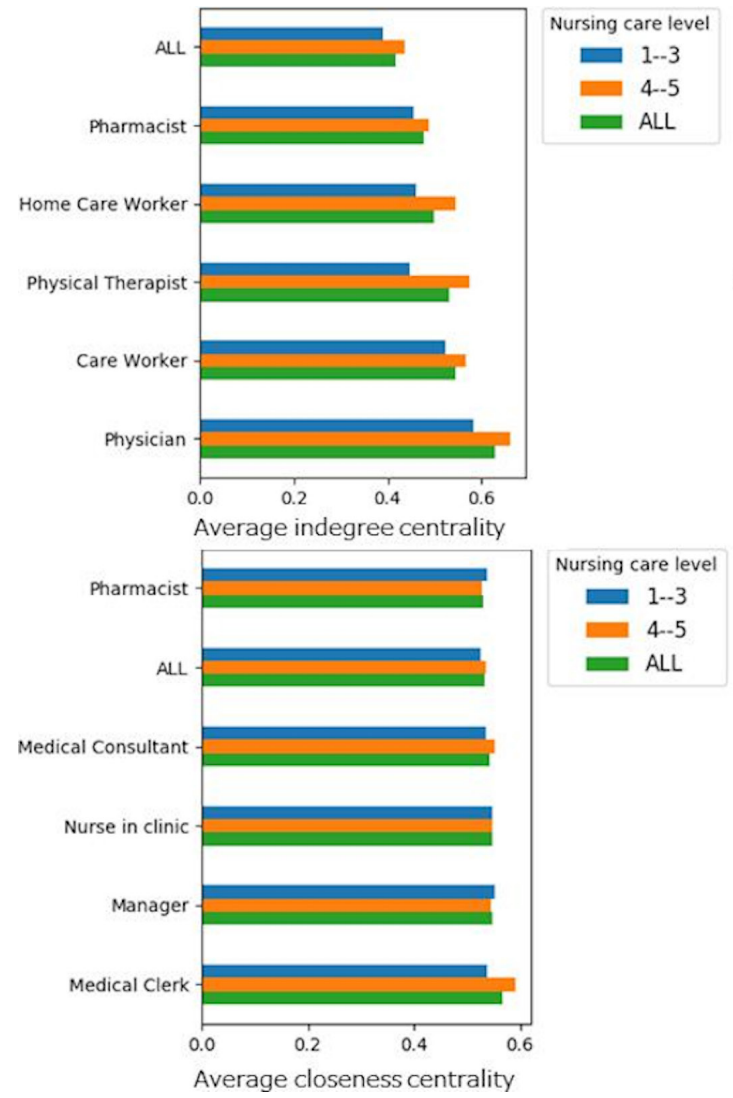

healthcare professionals across facilities that increasing nursing care level had relatively higher density, reciprocity and lower centrality, and involve different professions as central players.

Discussion of the findings in terms of density is difficult because this measure depends on the number of nodes. Nevertheless, density is a proposed factor for facilitating leadership in information sharing ${ }^{36}$ and, based on

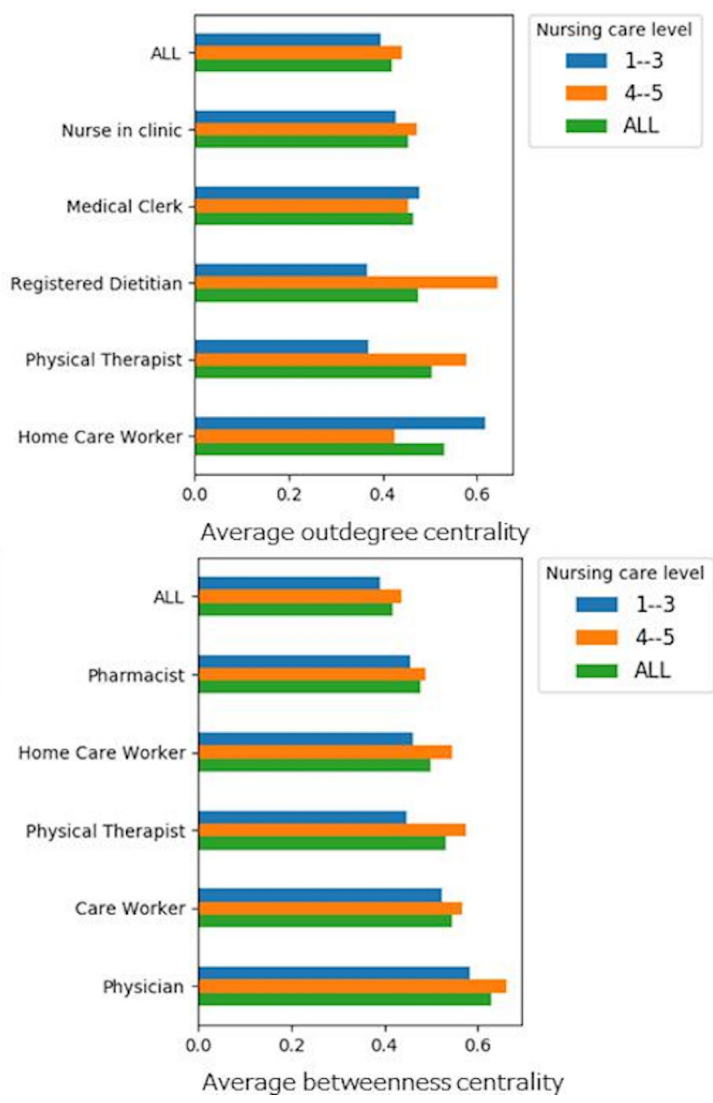

Figure 3 Differences in centrality among different professions between nursing care levels 1-3 and 4-5. 

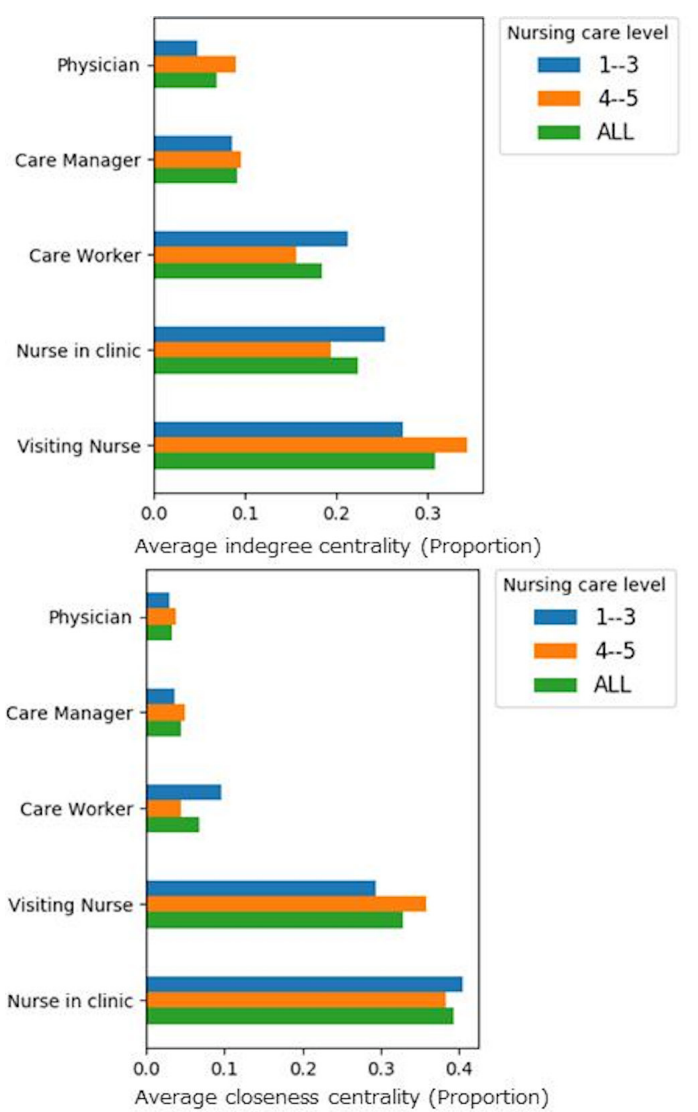

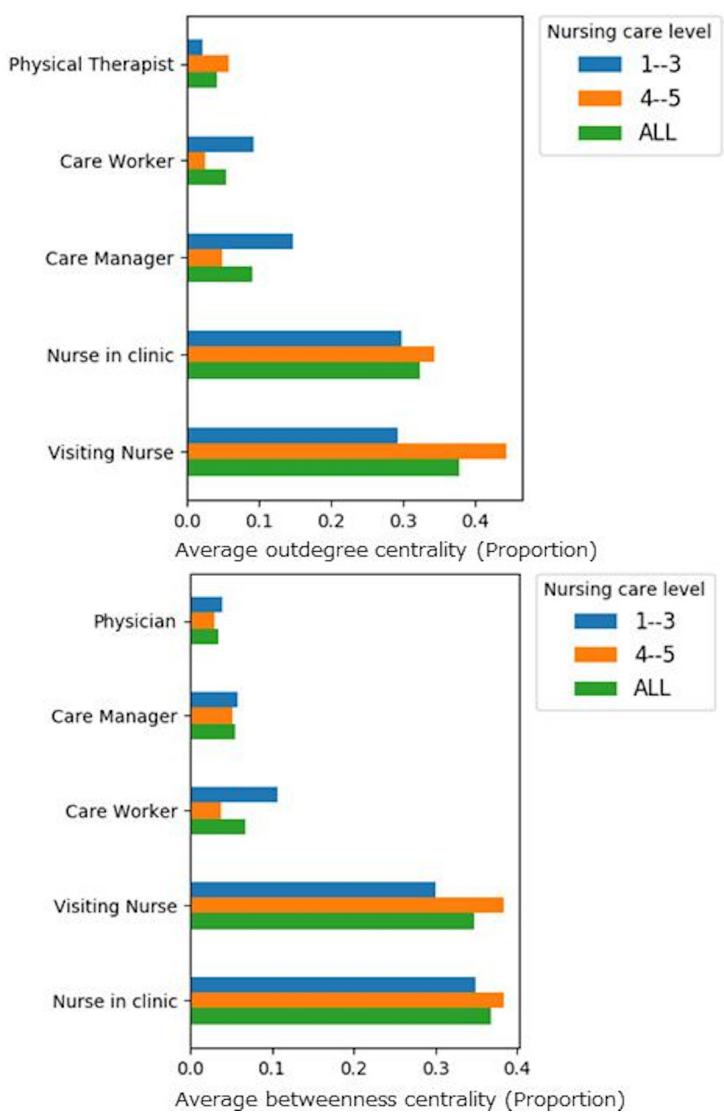

Figure 4 Differences in the number of highest ranking professionals between nursing care levels 1-3 and 4-5.

findings that density is positively correlated with group performance, ${ }^{3738}$ is a commonly used measure of network structure in a team. ${ }^{39}$ While such studies are scarce, a density of 0.4 in a network of nodes with an average of 18 healthcare professionals may be a criterion for future research.

In contrast, reciprocity is useful for capturing bidirectional relationships because it indicates whether the two parties involved in a relationship equally characterise the relationship. While the reciprocity of a network of professionals receiving advice on medication in emergencies was previously reported to be $0.28,{ }^{24}$ the present study showed a higher score of 0.4 . This finding may suggest functional differences between emergency departments, in which roles are clearly allocated to each individual profession, and home care networks in communitybased integrated care system, ${ }^{40}$ where all professions have comprehensive roles. This system, which the government of Japan spread nationwide, integrates a community's healthcare resources, through the coordination not only of outpatient and inpatient sections in hospitals or clinics, but also of welfare facilities, home-visit care services, and even mutual support activities among neighbourhoods. ${ }^{40}$

Centrality measures that allow network characterisation regardless of the number of nodes (degree-based, closeness, betweenness centrality) are widely used in actual analyses. Centrality provides an estimate of the activity and leadership level of a person or community with a central role in the community. ${ }^{41}$ This study demonstrated that the centrality of professional networks involving patients requiring heavier care tended to be lower than those involving patients requiring lighter care. This indicates that a variety of healthcare professions may play a leadership or central role in networks with heavier care requirement. In networks where patients seek advice on medication, nurses and physicians show low levels of communication, while pharmacists are highly centric. ${ }^{42}$ In this study, healthcare professions that read messages who have high indegree centrality differed from those that sent messages who have high outdegree centrality. This finding may suggest that the professional role of physicians may require them to provide specific information while home care workers and physical therapists tend to engage in taking care of patients through the exchange of information among multiple healthcare professionals.

Additionally, closeness centrality is the potential independence of a node in the flow of an information-sharing clinical network, and betweenness centrality is a centrality measure based on the degree by which a node mediates the relationship of other nodes. Nurses in the clinic and visiting nurses had higher ranked closeness centrality and betweenness centrality than other professionals (figure 4), while medical clerks, physicians, physical therapists and registered dieticians showed somewhat higher than average centrality measures for all professional networks (figure 3). This suggests that the relatively lower number of medical clerks, physicians, physical therapists and registered dieticians than visiting nurses and nurses in the 
clinic may have contributed to the difference between the average centrality measures and professions with highest ranked centrality. The number of physicians, physical therapists and registered dieticians among professionals who actively viewed and posted in the network may have affected the average centrality measures. Additionally, while physicians, physical therapists and registered dieticians as clear experts and medical clerks as economic and institutional experts serve as mediators regardless of the degree of care required, nurses which were highest ranked in centrality are expected to play a central role in mediating the relationships of patients using home medical care and care services. Previous findings have shown that hierarchical relationships in the healthcare domain, as revealed by centrality, may be a major problem for teams. ${ }^{17}$ Multiple daily communications within a team reportedly promote the development of trust among team members playing different roles, ${ }^{43}$ and promote energetic and emotional involvement, which are indispensable for task adjustment. ${ }^{44}$

Furthermore, while visiting nurses and nurses in the clinic are key players in networks the centrality roles of these professions differed only slightly between nursing care levels 1-3 and levels 4-5. After accounting for the fact that communication levels vary depending on the objective of the community, these results suggest that in City X, visiting nurses and nurses in the clinic, who have greater awareness of the severity of diseases/disorders, may play leadership roles in home care support networks comprising users requiring nursing care levels 4-5 (higher severity of disease/disorder). In particular, visiting nurses, who on their own initiative can conduct condition assessments at home and provide home care required for a particular disorder, seem more likely to play central roles in home care networks with high medical care dependence, such as those for terminal patients and patients with cancer. Meanwhile, based on findings for closeness centrality and betweenness centrality, nurses in the clinic likely play the role of a hub in multiprofessional networks. Further qualitative investigation is needed to determine the influence of networks on who play central roles, and how they do so, on the members within the network. Additional data are needed from studies examining associations with patient outcomes, surveys in other regions and qualitative surveys for verification. As a strength, to our knowledge, few studies have investigated virtual interprofessional interactions in community healthcare services using SNA. Thus, we consider that the patterns identified in the virtual interprofessional interaction in SNS are useful for developing SNS-based networks with multiple professionals in a community.

The study had several potential limitations. First, physicians might provide advice to nurses using tools (such as orally or via paper notes) other than SNS. The Japanese healthcare system, in which patients and their families call visiting nurses first, rather than physicians, may have affected the findings. The study participants may not be representative of Japan. Additionally, we did not clarify how network structures were associated with team function or healthcare outcomes. On the other hand, studies have reported that frequent sharing of patient/user information among team members is highly significant, and that clarifying the roles of various professions on the basis of interprofessional mutually dependent relationships is closely associated with patient outcomes. ${ }^{45}$ Previous studies using SNA have discussed health behaviours, adoption of evidence-based preventive interventions, social networks and cost effectiveness in cardiovascular teams. ${ }^{46}$ A study reported that $20 \%$ of teamwork-related team performance appeared as network differences. ${ }^{47}$ Given the lack of evidence on SNA in IPC in a community, the findings suggest that SNA has the potential to contribute to improving the provision of patient care and aid in the proposal of a highly cost-effective method. Further studies are also needed to determine the relationship between such networks and the successful achievement of good patient outcomes, cost effectiveness and other factors.

\section{CONCLUSION}

This study explored the network structure of SNS-based information-sharing networks for healthcare professionals across facilities that increasing nursing care level had relatively higher density, reciprocity and lower centrality, and involve different professions as central players. in one community in Japan. Associations between network structures and patient outcomes, cost effectiveness and other factors warrant further investigation.

Acknowledgements The authors sincerely appreciate Takumi Komorita, who provided data through "MeLL+ community".

Contributors All authors were involved in the conception and design of this study, carried out all qualitative enquiries, analysed the data and wrote the paper.

Funding This work was supported by JSPS KAKENHI Grant-in-Aid for Young Scientists (B) Grant Number JP19K19377.

Competing interests None declared.

Patient consent for publication Not required.

Ethics approval Ethics Committee of the Faculty of Medicine, University of Tsukuba (No. 1377)

Provenance and peer review Not commissioned; externally peer reviewed.

Data availability statement The datasets generated during and/or analysed during the current study are available from the corresponding author on reasonable request.

Open access This is an open access article distributed in accordance with the Creative Commons Attribution Non Commercial (CC BY-NC 4.0) license, which permits others to distribute, remix, adapt, build upon this work non-commercially, and license their derivative works on different terms, provided the original work is properly cited, appropriate credit is given, any changes made indicated, and the use is non-commercial. See: http://creativecommons.org/licenses/by-nc/4.0/.

ORCID iD

Junji Haruta http://orcid.org/0000-0003-4176-7665

\section{REFERENCES}

1 Muramatsu N, Akiyama H. Japan: super-aging Society preparing for the future. Gerontologist 2011;51:425-32. 
2 Arai H, Ouchi Y, Toba K, et al. Japan as the front-runner of superaged societies: perspectives from medicine and medical care in Japan. Geriatr Gerontol Int 2015;15:673-87.

3 World Health Organization. Strengthening of palliative care as a component of integrated treatment throughout the life course. J Pain Palliat Care Pharmacother 2014;28:130-4.

4 Thistlethwaite JE, Forman D, Matthews LR, et al. Competencies and frameworks in interprofessional education: a comparative analysis. Acad Med 2014;89:869-75.

5 Foronda C, MacWilliams B, McArthur E. Interprofessional communication in healthcare: an integrative review. Nurse Educ Pract 2016;19:36-40.

6 Haruta J, Yoshida K, Goto M, et al. Development of an interprofessional competency framework for collaborative practice in Japan. J Interprof Care 2018;32:436-43.

7 Espinoza P, Peduzzi M, Agreli HF, et al. Interprofessional team member's satisfaction: a mixed methods study of a Chilean Hospital. Hum Resour Health 2018;16:30.

8 Evans BM, Chi EH. An elaborated model of social search. Inf Process Manag 2010;46:656-78.

9 Morikawa M. Towards community-based integrated care: trends and issues in Japan's long-term care policy. Int $J$ Integr Care 2014;14:e005.

10 Masao Iwagami NT. The long-term care insurance system in Japan: past, present, and future. Jma J 2019;2:67-9.

11 Jippes E, Achterkamp MC, Brand PLP, et al. Disseminating educational innovations in health care practice: training versus social networks. Soc Sci Med 2010;70:1509-17.

12 Barnett S, Jones SC, Bennett S, et al. General practice training and virtual communities of practice - a review of the literature. BMC Fam Pract 2012:13:87.

13 Deng L, Poole MS. Learning through ICT-enabled social networks. IJITM 2008;7:374.

14 Winick $\mathrm{C}$. The diffusion of an innovation among physicians in a large City. Sociometry 1961;24:384

15 Coleman J, Katz E, Menzel H. The diffusion of an innovation among physicians. Sociometry 1957;20:253.

16 Chambers D, Wilson P, Thompson C, et al. Social network analysis in healthcare settings: a systematic scoping review. PLoS One 2012;7:e41911.

17 Bae S-H, Nikolaev A, Seo JY, et al. Health care provider social network analysis: A systematic review. Nurs Outlook 2015;63:566-84.

18 Benton DC, Pérez-Raya F, Fernández-Fernández MP, et al. A systematic review of nurse-related social network analysis studies. Int Nurs Rev 2015;62:321-39.

19 Rivera MT, Soderstrom SB, Uzzi B. Dynamics of dyads in social networks: assortative, relational, and proximity mechanisms. SSRN 2010.

20 van Beek APA, Wagner C, Spreeuwenberg PPM, et al. Communication, advice exchange and job satisfaction of nursing staff: a social network analyses of 35 long-term care units. BMC Health Serv Res 2011:11:140.

21 Vanderveen KA, Paterniti DA, Kravitz RL, et al. Diffusion of surgical techniques in early stage breast cancer: variables related to adoption and implementation of sentinel lymph node biopsy. Ann Surg Oncol 2007;14:1662-9.

22 Bradley F, Ashcroft D, Crossley N. Using social network analysis to study integration between general practitioners and community pharmacists: a novel application. Int J Pharm Pract 2010;4:3-4.

23 Rangachari $\mathrm{P}$, Rissing $\mathrm{P}$, Wagner $\mathrm{P}$, et al. A baseline study of communication networks related to evidence-based infection prevention practices in an intensive care unit. Qual Manag Health Care 2010.
24 Creswick N, Westbrook JI, Braithwaite J. Understanding communication networks in the emergency department. BMC Health Serv Res 2009;9:247-56.

25 Boyer L, Belzeaux R, Maurel O, et al. A social network analysis of healthcare professional relationships in a French Hospital. Int $J$ Health Care Qual Assur 2010;23:460-9.

26 O'Malley AJ, Marsden P V. The analysis of social networks. Heal Serv Outcomes Res Methodol 2008.

27 Cunha T, Tan C, Jurgens D, et al. Are all successful communities alike? Characterizing and predicting the success of online communities. In: The Web Conference 2019 - Proceedings of the World Wide Web Conference, 2019. Available: WWW 2019

28 Tsugawa S, Niida S. The impact of social network structure on the growth and survival of online communities. Vancouver, Canada: Association for Computing Machinery, 2019.

29 Hawe P, Webster C, Shiell A. A glossary of terms for Navigating the field of social network analysis. J Epidemiol Community Health 2004;58:971-5.

30 Costa LdaF, Rodrigues FA, Travieso G, et al. Characterization of complex networks: a survey of measurements. Advances in Physics 2007;56:167-242.

31 Newman MEJ. The structure and function of complex networks. SIAM Rev. 2003;45:167-256.

32 Latora V, Marchiori M. Efficient behavior of small-world networks. Phys Rev Lett 2001;87:198701.

33 Masuda N, Sakaki M, Ezaki T, et al. Clustering coefficients for correlation networks. Front Neuroinform 2018;12:7.

34 Freeman LC. Centrality in social networks conceptual clarification. Soc Networks 1978;1:215-39.

35 Scott J. Social network analysis: a Handbook. 2nd ed. Oaks, Thousand, CA: Sage, 2000.

36 Carson JB, Tesluk PE, Marrone JA. Shared leadership in teams: an investigation of antecedent conditions and performance. Acad Manag J 2007;50:1217-34.

37 Wong Sze-Sze, Wong SS. Task knowledge overlap and knowledge variety: the role of advice network structures and impact on group effectiveness. J Organ Behav 2008;29:591-614.

38 Lin Z, Yang H, Arya B, et al. Structural versus individual perspectives on the dynamics of group perfomance: theoretical exploration and empirical investigation. J Manage 2005;31:354-80.

39 Henttonen K. Exploring social networks on the team level-A review of the empirical literature. Journal of Engineering and Technology Management 2010;27:74-109.

40 Morikawa M. Towards community-based integrated care: Trends and issues in Japan's long-term care policy. Int $\mathrm{J}$ Integr Care 2014;26:e005.

41 Borgatti SP, Cross R. A relational view of information seeking and learning in social networks. Manage Sci 2003;49:432-45.

42 Creswick N, Westbrook Jl. Social network analysis of medication advice-seeking interactions among staff in an Australian hospital. Int J Med Inform 2010;79:e116-25.

43 Baker DP, Day R, Salas E. Teamwork as an essential component of high-reliability organizations. Health Serv Res 2006;41:1576-98.

44 Quinn RW, Dutton JE. Coordination as Energy-in-Conversation. AMR 2005;30:36-57.

45 Gittell JH, Godfrey M, Thistlethwaite J. Interprofessional collaborative practice and relational coordination: improving healthcare through relationships. J Interprof Care 2013;27:210-3.

46 Mundt MP, Gilchrist VJ, Fleming MF, et al. Effects of primary care team social networks on quality of care and costs for patients with cardiovascular disease. Ann Fam Med 2015;13:139-48.

47 Salas E, Cooke NJ, Rosen MA. On teams, teamwork, and team performance: discoveries and developments. Hum Factors 2008;50:540-7. 